\title{
On the complexity of Wafer-to-Wafer Integration
}

\author{
G. Duvillié ${ }^{1}$, M. Bougeret ${ }^{1}$, V. Boudet ${ }^{1}$, \\ T. Dokka ${ }^{2}$, R. Giroudeau ${ }^{1}$ \\ 1 LIRMM, Université Montpellier 2, France \\ \{guillerme.duvillie, vincent.boudet, marin.bougeret, rodolphe.giroudeau\}@lirmm.fr, \\ 2 Dept. of Management Science, Lancaster University \\ t.dokka@lancaster.ac.uk
}

\begin{abstract}
In this paper we consider the Wafer-to-Wafer Integration problem. A wafer is a $p$-dimensional binary vector. The input of this problem is described by $m$ disjoints sets (called "lots"), where each set contains $n$ wafers. The output of the problem is a set of $n$ disjoint stacks, where a stack is a set of $m$ wafers (one wafer from each lot). To each stack we associate a $p$-dimensional binary vector corresponding to the bit-wise AND operation of the wafers of the stack. The objective is to maximize the total number of " 1 " in the $n$ stacks. We provide $O\left(m^{1-\epsilon}\right)$ and $O\left(p^{1-\epsilon}\right)$ non-approximability results even for $n=2$, as well as a $\frac{p}{r}$-approximation algorithm for any constant $r$. Finally, we show that the problem is FPT when parameterized by $p$, and we use this FPT algorithm to improve the running time of the $\frac{p}{r}$-approximation algorithm.
\end{abstract}

\section{Introduction}

\subsection{Problem definition}

In this paper we consider Wafer-to-Wafer Integration problems. In these problems, we are given $m$ disjoint sets $V^{1} \ldots V^{m}$, where each set $V^{i}$ contains $n$ binary $p$-dimensional vectors. For any $j \in[n]_{1}{ }^{1}$, and any $i \in[m]_{1}$, we denote by $v_{j}^{i}$ the $j^{t h}$ vector of set $V^{i}$, and for any $k \in[p]_{1}$ we denote by $v_{j}^{i}(k) \in\{0,1\}$ the $k^{t h}$ component of $v_{j}^{i}$.

Let us now define the output. A stack $s=\left(v_{1}^{s}, \ldots, v_{m}^{s}\right)$ is an $m$-tuple of vectors such that $v_{i}^{s} \in V^{i}$, for any $i \in[m]_{1}$. An output of the problem is a set $S=\left\{s_{1}, \ldots, s_{n}\right\}$ of $n$ stacks such that for any $i$ and $j$, vector $v_{j}^{i}$ is contained exactly in one stack. An example of input and output is depicted in Figure 1.

These problems are motivated by an application in IC manufacturing in semiconductor industry, see [10] for more details about this application. A wafer can be seen as a string of bad dies (0) and good dies (1). Integrating two wafers corresponds to superimposing the two corresponding strings. In this operation, a position in the merged string is only 'good' when the two corresponding dies are good, otherwise it is 'bad'. The objective of Wafer-to-Wafer Integration is to form $n$ stacks, while maximizing the overall quality of the stacks (depending on the objective function).

\footnotetext{
${ }^{1}$ The notation $[n]_{j}$ stands for $\{j, \ldots, n\}$.
} 
Let us now define several objective functions, and the corresponding optimization problems. We consider the operator $\wedge$ which maps two $p$-dimensional vectors to another one by performing the logical and operation on each component of entry vectors. More formally, given two $p$-dimensional vectors $u$ and $v$, we define $u \wedge v=(u(1) \wedge v(1), u(2) \wedge v(2), \ldots, u(p) \wedge v(p))$. We associate to any stack $s=\left(v_{1}^{s}, \ldots, v_{m}^{s}\right)$ a binary $p$-dimensional vector $v_{s}=\bigwedge_{i=1}^{m} v_{i}^{s}$. Then, the profit of a stack $s$ is given by $c\left(v_{s}\right)$, where $c(v)=\sum_{k=1}^{p} v(k)$. Roughly speaking, the profit of a stack is the number of good bits in the stack, where a good bit (in position $k$ ) survives iff all the vectors of the stack have a good bit in position $k$.

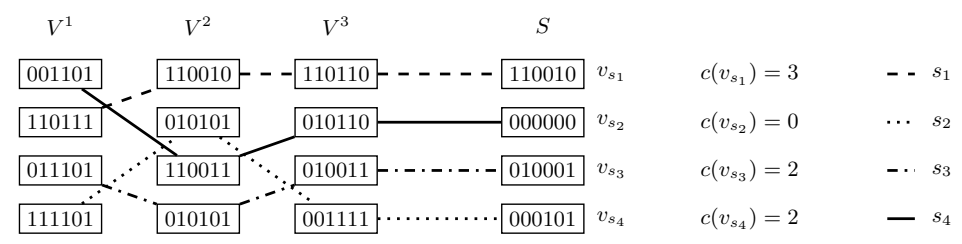

Fig. 1. Example of $\max \sum 1$ instance with $m=3, n=4, p=6$ and of a feasible solution $S$ of profit $f_{\Sigma 1}(S)=7$.

We are now ready to define two following optimization problems:

\begin{tabular}{ll}
\hline Set of problems $1 \max \sum 1$ and $\min \sum 0$ \\
\hline Input & $m$ sets of $n$ binary $p$-dimensional vectors \\
Output & a set $S$ of $n$ disjoint stacks \\
Objective & $\max \sum 1:$ maximize $f_{\sum 1}(S)=\sum_{j=1}^{n} c\left(v_{s_{j}}\right)$, the total number of good bits \\
functions & $\min \sum 0:$ minimize $f_{\sum 0}(S)=n p-\sum_{j=1}^{n} c\left(v_{s_{j}}\right)$, the number of bad bits \\
\hline
\end{tabular}

Instance of these problems will be denoted by $I[m, n, p]$. The notation $f(S)$ (instead of $f_{\sum 0}(S), f_{\sum 1}(S), \ldots$ ) will be used when the context is non ambiguous.

\subsection{Related work}

In this paper we consider results in the framework of approximation and fixed parameter tractability theory. We only briefly recall the definitions here and refer the reader to $[9,11]$ for more information. For any $\rho>1$, a $\rho$-approximation algorithm $A$ (for a maximization problem) is such that for any instance $I$, $A(I) \geq \frac{O p t(I)}{\rho}$, where $O p t(I)$ denotes the optimal value. The input of a parameterized (decision) problem $\Pi$ is a couple $(X, \kappa)$, where $X \subseteq \Sigma^{*}$ is a classical decision problem, and $\kappa: \Sigma^{*} \longrightarrow \mathbb{N}$ is a parameterization. Deciding $\Pi$ requires to determine for any instance $I \in \Sigma^{*}$ if $I \in X$. Finally, we say that an algorithm $A$ decides $\Pi$ in FPT time (or that $\Pi$ is FPT parameterized by $\kappa$ ) iff there exists a computable function $f$ and a constant $c$ such that for any $I, A(I)$ runs in $\mathcal{O}\left(f(\kappa(I))|I|^{c}\right)$. 
The max $\sum 1$ problem was originally defined in [10] as the "yield maximization problem in wafer-to-wafer 3-D integration technology". Authors of [10] point out that "the classical NP-hard 3-D matching problem is reducible to the max $\sum 1$ problem". However, they do not provide the reduction and they only conclude that $\max \sum 1$ is NP-hard without stating consequences on the approximability. They also notice that $\max \sum 1$ is polynomial for $m=2$ (as it reduces to finding a maximum profit perfect matching in a bipartite graph, solved by Hungarian Method), and design the "iterative matching heuristic" (IMH) that computes a solution based on (2D) matchings.

In [3] and [4] we investigated the min $\sum 0$ problem by providing a $\frac{4}{3}-$ approximation algorithm for $m=3$ and several $f(m)$-approximation algorithms for arbitrary $m$ (and for a more general profit function $c$ ). Furthermore, we also noticed in [3] that the natural ILP formulation implied that $\min \sum 0$ and $\max \sum 1$ are polynomial for fixed $p$. Concerning negative results, the implicit straightforward reduction from k-Dimensional MATCHING in [3] and made explicit in [5], shows that $\min \sum 0$ is NP-hard, and $\max \sum 1$ is $\mathcal{O}\left(\frac{m}{\ln m}\right)$ non-approximable. The more complex reduction of [4] shows that $\min \sum 0$ is APX-hard even for $m=3$, and thus is very unlikely to admit a PTAS.

\begin{tabular}{|c|c|c|c|}
\hline & {$[10]$} & {$[3],[4]$} & This paper \\
\hline $\max \sum 1$ & NP-hard & $\mathcal{O}\left(\frac{m}{\ln m}\right)$ inapproximability & $\begin{array}{c}\text { for any } \varepsilon, \mathcal{O}\left(p^{1-\varepsilon}\right) \text { and } \mathcal{O}\left(m^{1-\varepsilon}\right) \\
\text { inapproximability (even for } n=2) \\
\frac{p}{r} \text {-approximation in } \\
\mathcal{O}(f(r) \text { poly }(m+n+p)) \\
\text { pPT } / p\end{array}$ \\
\hline $\min \sum 0$ & & $\begin{array}{c}\text { for } m=3: \frac{4}{3} \text {-approximation, APX-hard } \\
f(m) \text {-approximation for general } m \\
\text { polynomial for fixed } p\end{array}$ & FPT $/ p$ \\
\hline
\end{tabular}

Table 1. Overview of results on Wafer-to-Wafer Integration

\subsection{Contributions}

In this paper we mainly study the max $\sum 1$ problem, with a particular focus on parameter $p$. We prove in Subsection 2.2 that even for $n=2$, for any $\epsilon$, there is no $\rho(m, p)$-approximation algorithm for $\max \sum 1$ such that $\rho(x, x)=\mathcal{O}\left(x^{1-\epsilon}\right)$ unless $\mathbf{P}=\mathbf{N P}$ (this implies in particular no $\mathcal{O}\left(p^{1-\epsilon}\right)$ and no $\mathcal{O}\left(m^{1-\epsilon}\right)$ ratios). These negative results show that the simple $p$-approximation presented in Section 3.1 is somehow the best ratio we can hope for. Nevertheless, looking for better positive results we focus on $\frac{p}{r}$-approximation algorithm for any constant $r$. It turns out that any $\mathcal{O}(f(n, m, p))$ exact algorithm for $\max \sum 1$ can be used to derive a $\frac{p}{r}$-approximation in $\mathcal{O}(p \times f(n, m, r))$. This motivates our main result: determining the complexity of the $\max \sum 1$ problem when parameterized by $p$. The natural ILP in [4] implied that $\max \sum 1$ (and $\min \sum 0$ ) is polynomial for fixed $p$. In Section 3.2, we improve this result by showing that $\max \sum 1$ (and $\min \sum 0$ ) are FPT parameterized by $p$. 


\section{$2 \quad$ Negative Results}

In order to obtain negative results for $\max \sum 1$, let us first introduce two related problems defined in the table Set of Problems 2.

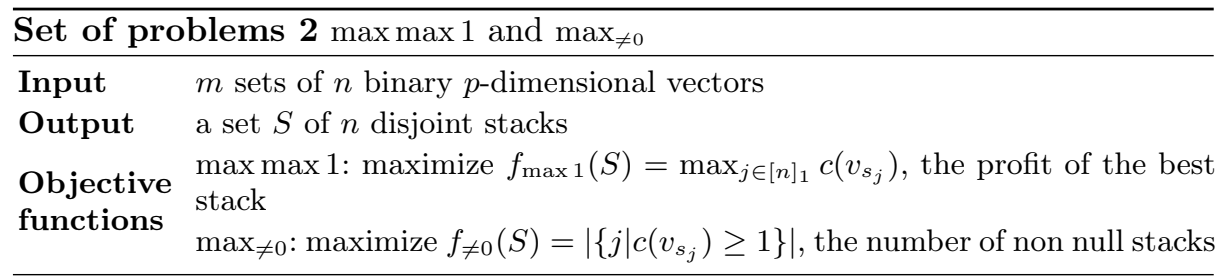

Roughly speaking, we will see that approximating max $\sum 1$ is harder than approximating these two problems, and that these problems are themselves nonapproximable.

To show that approximability is preserved we will provide strict reductions [1]. Indeed, if there is a strict reduction from $\Pi_{1}$ to $\Pi_{2}$, then any polynomial $\rho$ approximation for $\Pi_{2}$ yields to a $\rho$-approximation for $\Pi_{1}$. Notice that in the following we will rather provide reductions as defined in the following property.

Property 1 Let $\Pi_{1}$ and $\Pi_{2}$ be two maximization problems with their given objective functions $m_{1}$ and $m_{2}$. Let $f$ be a polynomial function that given any instance $x$ of $\Pi_{1}$ associate an instance $f(x)$ of $\Pi_{2}$. Let $g$ be a polynomial function that given any instance $x$ of $\Pi_{1}$, and feasible solution $S_{2}$ of $f(x)$, associates a feasible solution $g\left(x, S_{2}\right)$ of $\Pi_{1}$. If $f$ and $g$ verify the two following conditions:

1. $O p t(x)=O p t(f(x))$

2. $m_{1}\left(g\left(x, S_{2}\right)\right) \geq m_{2}(f(x))$

then $(f, g)$ is a strict reduction.

\subsection{Relation between $\max _{\neq 0}, \max \max 1$ and $\max \sum 1$}

Observation 1 There exists a strict reduction from $\max \max 1$ to $\max \sum 1$.

Proof. Let us construct $(f, g)$ as in Property 1. Consider an instance $I^{\prime}\left[m^{\prime}, n^{\prime}, p^{\prime}\right]$ of $\max \max 1$. We construct an instance $I[m, n, p]$ of $\max \sum 1$ as follows: we set $p=p^{\prime}, n=n^{\prime}, m=m^{\prime}+1$. The $m^{\prime}$ sets of $I^{\prime}\left[m^{\prime}, n^{\prime}, p^{\prime}\right]$ remain unchanged in $I[m, n, p]: \forall i \in\left[m^{\prime}\right]_{1}, V^{i}=V^{\prime i}$ and the last set $V^{m^{\prime}+1}$ contains $(n-1)$ "zero vectors" (i.e. vectors having only 0) and one "one vector" (i.e. vector having only 1).

Informally, the set $V^{m^{\prime}+1}$ of $I$ behaves like a selecting mask: since all stacks except one are turned into zero stacks when assigning the vectors of last set, the unique "one vector" of set $V^{m^{\prime}+1}$ must be assigned to the best stack, and maximizing the sum of the stacks is equivalent to maximizing the best stack.

More precisely, it is straightforward to see that the following statement is true: $\forall x, \exists$ solution $S^{\prime}$ of $\max \max 1$ of value $f_{\max 1}\left(S^{\prime}\right)=x \Leftrightarrow \exists$ solution $S$ of 
$\max \sum 1$ of value $f_{\sum 1}(S)=x$. Thus, we get $O p t_{\max \max 1}\left(I^{\prime}\right)=O p t_{\max \sum 1}(I)$. As the previous reduction is polynomial, and a solution of $I^{\prime}$ can be deduced from a solution of $I$ in polynomial time, we get the desired result.

Observation 2 There exists a strict reduction from $\max _{\neq 0}$ to $\max \sum 1$.

We refer the reader to [5] for the reduction proving this lemma.

According to Observations 1 and 2, any non-approximability result for $\max _{\neq 0}$ or max max 1 will transfer to $\max \sum 1$. This motivates the next section.

\subsection{Hardness of $\max _{\neq 0}$ and $\max \max 1$}

The reduction from $\mathrm{k}$-Dimensional MATCHING $(\mathrm{kDM})$ provided in [3] can be adapted to $\max \sum 1$ instead of $\min \sum 0$ as shown in [5]. Unlike the case of $\min \sum 0$, the reduction preserves approximability:

Theorem 1 (implicit in [3]). There is a strict reduction from $k D M$ to $\max _{\neq 0}$.

As it is NP-hard to approximate $\mathrm{kDM}$ to a factor $\mathcal{O}\left(\frac{k}{\ln (k)}\right)$ [6], we get the following corollary:

Corollary 1. It is NP-hard to approximate $\max _{\neq 0}$ within a factor $\mathcal{O}\left(\frac{m}{\ln (m)}\right)$.

We can also notice that any $\frac{m}{r}$-approximation ratio (for a constant $r \geq 3$ ) for $\max _{\neq 0}$ or $\max \sum 1$ would improve the currently best known ratio for $\mathrm{kDM}$ set to $\frac{k+1+\epsilon}{3}$ in $[2]$.

Let us now consider a new reduction which provides results for $n=2$ and according to parameter $p$.

Theorem 2. There is a strict reduction from Maximum Clique Problem to $\max \max 1$ for $n=2$.

Proof. Let us construct $(f, g)$ as in Property 1. Let us consider an instance $G=(V, E)$ of the Maximum Clique Problem. The corresponding instance of $\max \max 1$ is constructed as follows. We consider $m=|V|$ sets, each having two vectors. All the vectors have $p=|V|$ bits. For each vertex $i$ of $V$, we create the set $V^{i}=\left(v_{1}^{i}, v_{2}^{i}\right)$. For any $i$, we define $v_{1}^{i}=\left(v_{1}^{i}(1), v_{1}^{i}(2), \ldots, v_{1}^{i}(p)\right)$, where $v_{1}^{i}(k)=1$ iff $\{i, k\} \in E$ or $i=k$, and $v_{2}^{i}=\left(v_{2}^{i}(1), v_{2}^{i}(2), \ldots, v_{2}^{i}(p)\right)$, where $v_{2}^{i}(k)=1$ iff $i \neq k$. In other words, $v_{1}^{i}$ corresponds to the $i^{t h}$ row of the adjacency matrix of $G$, with a self loop.

The idea is that selecting $v_{1}^{i}$ corresponds to selecting vertex $i$ in graph, and selecting $v_{2}^{i}$ will turn the $i^{\text {th }}$ component to 0 , which corresponds to a penalty for not choosing vertex $i$.

We first need to state an intermediate lemma. For any stack $s=\left\{v_{1}^{s}, \ldots, v_{m}^{s}\right\}$, let $X_{s}=\left\{i \mid v_{i}^{s}=v_{1}^{i}\right\}$ be the associated set of vertices in $G$. Recall that $v_{s}$ is the $p$ dimensional vector representing $s$.

Lemma 1. $\forall i \in[p]_{1}, v_{s}(i)=1 \Leftrightarrow\left(\left(i \in X_{s}\right)\right.$ and $\left.\left(\forall x \in X_{s} \backslash i,\{x, i\} \in E\right)\right)$.

$\triangle$ Let us first prove Lemma 1. Suppose $i^{\text {th }}$ component of $v_{s}$ is 1 . This implies that $v_{1}^{i} \in s$, and thus $i \in X_{s}$. Now, suppose by contradiction that $\exists x \in X_{s} \backslash i$ 
such that $\{x, i\} \notin E . x \in X_{s}$ implies that $v_{1}^{x} \in s$. Moreover, $v_{s}(i)=1$ implies that $v_{1}^{x}(i)=1$, and thus $\{x, i\} \in E$, which leads to a contradiction. Suppose now that $i \in X_{s}$, and $\forall x \in X_{s} \backslash i,\{x, i\} \in E$. Let us prove that $\forall i^{\prime}, v_{i^{\prime}}^{s}(i)=1$. Notice first that for $i^{\prime}=i$ we have $v_{i}^{s}(i)=v_{1}^{i}(i)=1$. Moreover, $\forall i^{\prime} \neq i$ such that $i^{\prime} \notin X_{s}$ we have $v_{i^{\prime}}^{s}(i)=v_{2}^{i^{\prime}}(i)=1$. Finally, $\forall i^{\prime} \neq i$ such that $i^{\prime} \in X_{s}$, we have $v_{i^{\prime}}^{s}(i)=v_{1}^{i^{\prime}}(i)=1$ as $\left\{i^{\prime}, i\right\} \in E . \triangle$

It is now straightforward to prove that $\forall x, " \exists$ solution $S$ for max max 1 of value $f_{\max 1}(S)=x \Leftrightarrow \exists$ a clique $X$ in $G$ of size $x$." Indeed, suppose first that we have a solution $S$ such that $f_{\max 1}(S)=x$. Let $s=\left(v_{1}^{s}, \ldots, v_{m}^{s}\right)$ be the stack in $S$ of value $x$, and let $G_{s}=\left\{k \mid v_{s}(k)=1\right\}$ be the set of good bits of $s$. We immediately get that the vertices corresponding to $G_{s}$ form a clique in $G$, as $\forall i$ and $j \in G_{s}$ the previous property implies that $i \in X_{s}, j \in X_{s}$, and thus $\{i, j\} \in E$. Suppose now that there is a clique $X^{*}$ in $G$, and let $s$ be a stack such that $X_{s}=X^{*}$. The previous property implies that $\forall i \in X_{s}, v_{s}(i)=1$.

Thus, $O p t_{\max \max 1}(I)$ is equal to the size of the maximum clique in $G$. As the previous reduction is polynomial, and as a solution of $S$ of $I$ can be translated back in polynomial time into the corresponding clique in $G$ (of same size), we get the desired result.

As for any $\epsilon$ there is no $\mathcal{O}\left(|V|^{1-\epsilon}\right)$-approximation for Maximum Clique ProbLEM (with set of vertices $V$ ) unless $\mathbf{P}=\mathbf{N P}$ [12], we get the following corollary:

Corollary 2. Even for $n=2$, for any $\epsilon$, there is no $\rho(m, p)$-approximation such that $\rho(x, x)=\mathcal{O}\left(x^{1-\epsilon}\right)$ for $\max \max 1$ and thus for $\max \sum 1$. Notice that in particular, $\mathcal{O}\left(p^{1-\epsilon}\right)$ and $\mathcal{O}\left(m^{1-\epsilon}\right)$ are not possible, but for example $(\mathrm{pm})^{\frac{1}{2}}$ is not excluded.

To summarize, the main negative results for $\max \sum 1$ are no $\mathcal{O}\left(p^{1-\epsilon}\right)$-approximation and no $\mathcal{O}\left(m^{1-\epsilon}\right)$ approximation for $n=2$, and no $\mathcal{O}\left(\frac{m}{\ln m}\right)$-approximation for arbitrary $n$ (using the reduction from k-Dimensional MATCHing of [4]). Notice that it does not seem obvious to adapt the previous reductions to provide the same non-approximability results for $\min \sum 0$. Thus, the question of improving the $f(m)$ ratios provided in [4] is still open.

\section{Positive Results}

In this Section, we develop some polynomial-time approximation algorithm for $\max \sum 1$. Then, we show that $\max \sum 1$ and $\min \sum 0$ are $\mathbf{F P T}$ parameterized by $p$.

\section{$3.1 \quad \frac{p}{r}$-approximation}

Given the previous negative results, it seems natural to look for ratio $\frac{p}{r}$, where $r$ is a constant. Let us first see how to achieve a ratio $p$ with Algorithm 1.

Property 1. Algorithm 1 is a $p$-approximation algorithm for $\max \sum 1$. 


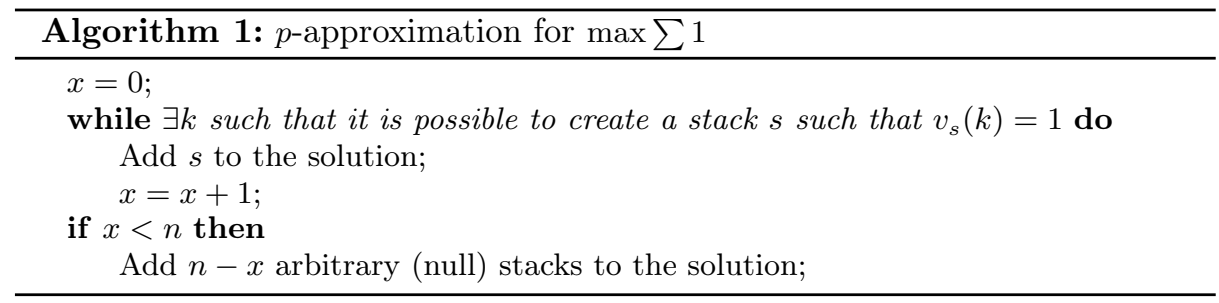

Proof. Let $S=S_{\neq 0} \bigcup S_{0}$ be the solution computed by the algorithm, where $S_{\neq 0}$ is the set of non zero stacks, and $S_{0}$ is the set of the remaining null stacks. Since $S_{\neq 0}$ and $S_{0}$ are disjoint, we have $S_{0}=S \backslash S_{\neq 0}$. Let $n_{1}=\left|S_{\neq 0}\right|$, and $\forall i$, let $V^{\prime i}=V^{i} \cap S_{0}$. Let $n_{2}=\left|S_{0}\right|=\left|V^{\prime i}\right|$ (all the $V^{\prime i}$ have the same size). Notice that $n=n_{1}+n_{2}$.

As the algorithm cannot create any non null stack at the end of the loop, we know that for any position $k \in[p]_{1}$, there is a set $i(k)$ such for any vector $w \in V^{\prime i(k)}, w(k)=0$. In other words, we can say that there is a column of $n_{2}$ zeros in set $V^{\prime i(k)}$. Notice there may be several columns of zeros in a given set. Thus, we deduce that there are at least $p$ columns (of $n_{2}$ zeros) in the vectors of $V^{\prime i(k)}$. Moreover, as none of these zeros can be matched in a solution, we know that these $n_{2} p$ zeros will appear in any solution.

Thus, given $S^{*}$ an optimal solution, we have $f\left(S^{*}\right) \leq n p-n_{2} p=n_{1} p$. As $f(S) \geq n_{1}$, we get the desired result.

Given a fixed integer $r$ (and targeting a ratio $\frac{p}{r}$ ), a natural way to extend Algorithm 1 is to first look for $r$ t-tuples (i.e. find $\left(k_{1}, \ldots, k_{r}\right)$ such that it is possible to create $s$ such that $\left.v_{s}\left(k_{1}\right)=\cdots=v_{s}\left(k_{r}\right)=1\right)$ ), then $(r-1)$ t-tuple, etc. However, even for $r=2$ this algorithm is not sufficient to get a ratio $\frac{p}{2}$, as shown by the example depicted in Figure2.

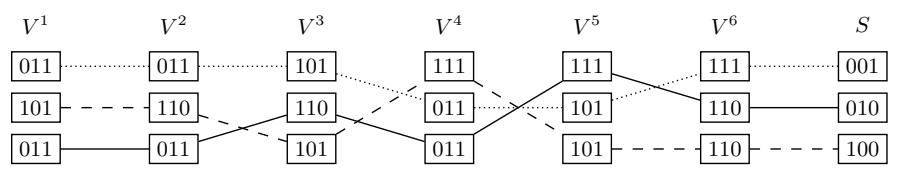

Fig. 2. Counter-example showing that Algorithm 1 for $r=2$ remains a $p$ approximation. The depicted stacks correspond to an optimal solution of profit 3 whereas the algorithm outputs a solution of profit 1 .

In this example it is not possible to create any stack of value strictly greater than 1 since set $V^{1}$ kills positions $\{1,2\}$ (we say that a set kills positions $\left\{k_{1}, k_{2}\right\}$ iff there is no vector in the set such that $w\left(k_{1}\right)=w\left(k_{2}\right)=1$ ), set $V^{2}$ kills positions $\{1,3\}$, and set $V^{3}$ kills positions $\{2,3\}$.

Thus, in this case (and more generally when no stack of value greater than 1 can be created), the solution computed by the algorithm for $r=2$ is the same as one computed by Algorithm 1. In the worst case, the algorithm creates only one stack of value 1 (by choosing the first vector of each set). However, as depicted in Figure 2, the optimal value is 3, and thus the ratio $\frac{p}{2}$ is not verified. 
In other words, knowing that no stack of profit 2 can be created does not provide better results for Algorithm 1. This motivates the different approach we follow hereafter.

Property 2. Suppose that there exists an exact algorithm for max $\sum 1$ running in $f(n, m, p)$. Then, for any $r \in[p]_{1}$ we have a $\frac{p}{r}$-approximation running in $\mathcal{O}(p \times$ $f(n, m, r))$.

Proof. The idea is to use a classical "shifting technique" by guessing the subset of the $r$ most valuable consecutive positions in the optimal solution, and run the exact algorithm on these $r$ positions.

Let $S^{*}$ be an optimal solution for $\max \sum 1$. Let us write $f\left(S^{*}\right)=\sum_{k=1}^{p} a_{k}$, where $a_{k}=\left|\left\{s \in S^{*} \mid v_{s}(k)=1\right\}\right|$ is the number of stacks in $S^{*}$ that save position $k . \forall k \in[p-1]_{0}$, let $X_{k}=\{k, \ldots,(k+r-1) \bmod p\}$, and $\sigma_{k}=\sum_{t \in X_{k}} a_{t}$. Notice that we have $\sum_{k=1}^{p} \sigma_{k}=r \sum_{k=1}^{p} a_{k}=r f\left(S^{*}\right)$, as each value $a_{k}$ appears exactly $r$ times in $\sum_{k=1}^{p} \sigma_{k}$. This implies $\max _{k} \sigma_{k} \geq \frac{r}{p} f\left(S^{*}\right)$.

For any $k$, let $I_{k}$ be the restricted instance where all the vectors are truncated to only keep positions in $X_{k}$ (there are still $n m$ vectors in $I_{k}$, but each vector is now a $r$ dimensional vector). By running the exact algorithm on all the $I_{k}$ and keeping the best solution, we get a $\frac{p}{r}$-approximation running in $\mathcal{O}(p f(n, m, r))$.

The previous lemma motivates the exact resolution of max $\sum 1$ in polynomialtime for fixed $p$. It is already proved in [4] that min $\sum 0$ can be solved in $\mathcal{O}\left(m\left(n^{2^{p}}\right)\right)$. As this result also apply to $\max \sum 1$, we get a $\frac{p}{r}$-approximation running in $\mathcal{O}\left(p m\left(n^{2^{r}}\right)\right)$, for any $r \in[p]_{1}$. Our objective is now to improve this running time by showing that $\max \sum 1$ (and $\min \sum 0$ ) are even FPT parameterized by $p$ (and not only polynomial for fixed $p$ ).

\subsection{Faster algorithm for fixed $p$ for $\max \sum 1$}

Definition 1. For any $t \in\left[2^{p}-1\right]_{0}$, we define configuration $t$ as $B_{t}$ : the $p$ dimensional binary vector that represents $t$ in binary. We say that a $p$-dimensional vector $v$ is in configuration $t$ iff $v=B_{t}$.

First ideas to get an FPT algorithm

Let us first recall our previous algorithm in [4] for fixed $p$. This result is obtained using an integer linear programming formulation of the following form. The objective function is $\min \sum_{t=0}^{2^{p}-1} x_{t} \bar{c}_{t}$ (recall that in [4] the considered objective function is $\left.\min \sum 0\right)$, where $x_{t} \in[n]_{0}$ is an integer variable representing the number of stacks in configuration $t$, and $\bar{c}_{t} \in[p]_{0}$ is the number of 0 in configuration $t$.

This is a good starting point to get an FPT algorithm. Indeed, if we note $n_{v a r}$ (resp. $m_{c t r}$ ) the number of variables (resp. number of constraints) of an ILP, for any $A \in \mathbb{Q}^{n_{\text {var }} \times m_{c t r}}, b \in \mathbb{Q}^{m_{c t r}}$, the famous algorithm of Lenstra [8] allows us to decide the feasibility of an ILP, under the form $\exists$ ? $x \in \mathbb{Z}^{n_{v a r}} \mid A x \leq b$, in time $\mathcal{O}\left(C^{n_{\text {var }}^{3}} m_{\text {ctr }}^{O(1)}\right)$ (this running time is given in [7]), where $C$ is a constant. Thus, to get an FPT algorithm parameterized by $p$, it is sufficient to write $\min \sum 0$ (and $\left.\max \sum 1\right)$ as an ILP using $f(p)$ variables. 
However, it remains now to add constraints that represent the min $\sum 0$ problem. In [4], these constraints are added using $z_{j t}^{i}$ variables (for $i \in[m]_{1}, j \in[n]_{1}, t \in$ $\left.\left.\left[2^{p}-1\right]_{0}\right)\right)$, where $z_{j t}^{i}=1$ iff $v_{j}^{i}$ is assigned to a stack of type $t$. Nevertheless these new $\mathcal{O}\left(m n 2^{p}\right)$ variables prevent us to use [8]. Thus, we now come back to the $\max \sum 1$ problem, and our objective is to express the constraints using only the $\left\{x_{t}\right\}$ variables.

Presentation of the new ILP for $\max \sum 1$

For any $t \in\left[2^{p}-1\right]_{0}$, we define an integer variable $x_{t} \in[n]_{0}$ representing the number of stacks in configuration $t$. Let also $c_{t} \in[p]_{1}=c\left(B_{t}\right)$ be the number of 1 in configuration $t$.

Definition 2. A profile is a tuple $P=\left\{x_{0}, \ldots, x_{2^{p}-1}\right\}$ such that $\sum_{t=0}^{2^{p}-1} x_{t}=n$.

Definition 3. The profile $\operatorname{Pr}(S)=\left\{x_{0}, \ldots, x_{2^{p}-1}\right\}$ of a solution $S=\left\{s_{1}, \ldots, s_{n}\right\}$ is defined by $x_{t}=\mid\left\{i \mid v_{s_{i}}\right.$ is in configuration $\left.t\right\} \mid$, for $t \in\left[2^{p}-1\right]_{0}$.

Definition 4. Given a profile $P$, an associated solution $S$ is a solution such that $\operatorname{Pr}(S)=P$. We say that a profile $P$ is feasible iff there exists an associated solution $S$ that is feasible.

Notice that the definition of associated solutions also applies to a non feasible profile. In this case, any associated solution will also be non feasible.

Obviously, the $\max \sum 1$ problem can be formulated using the following ILP:

$$
\begin{aligned}
\max \sum_{t=0}^{2^{p}-1} x_{t} c_{t} \quad \text { subject to } & \sum_{t=0}^{2^{p}-1} x_{t}=n \\
& \forall 0 \leq t<2^{p}, x_{t} \in \mathbb{N} \\
& P=\left\{x_{t}\right\} \text { is feasible }
\end{aligned}
$$

Our objective is now to express the feasibility of a profile by using only these $2^{p}$ variables. Roughly speaking, the idea to ensure the feasibility is the following. Let us suppose (with $p=2$ and $n=4$ for example) that there exists a feasible solution of fixed profile $x_{0}=0, x_{1}=1, x_{2}=2, x_{3}=1$. Suppose also that the first set is as depicted in Figure 3. To create a feasible solution with this profile, we have to "satisfy" (for each set $V^{i}$ ) the demands $x_{t}$ for all configurations $t$. For example in set 1 , the demand $x_{2}$ can be satisfied by using one vector in configuration 2 and one vector of configuration 3, and the demand 3 can be satisfied using the remaining vector of 3 (the demand $x_{0}$ is clearly satisfied). Notice that a demand of a given configuration (e.g. configuration 2 here) can be satisfied using a vector that "dominates" this configuration (e.g. configuration 3 here). The notion of domination will be introduced in Definition 5 . Thus, a feasible profile implies that for any set $i$ there exists a perfect matching between the vectors of $V^{i}$ and the profile $\left\{x_{t}\right\}$.

Let us now define more formally the previous ideas.

\section{Definition 5 (Domination).}

A p-dimensional vector $v_{1}$ dominates a p-dimensional vector $v_{2}$ (denoted by $\left.v_{1} \gg v_{2}\right)$ iff $\forall k \in[p]_{1}, v_{2}(k)=1 \Rightarrow v_{1}(k)=1$. 


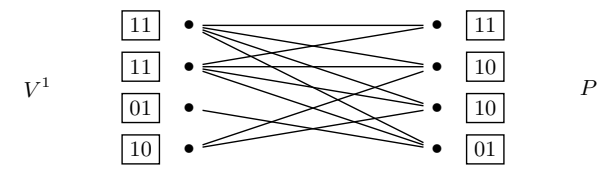

Fig. 3. Example showing that satisfying demands of profile $P$ with set 1 requires to find a perfect matching. Edges represent domination between configuration.

A configuration $t_{1} \in\left[2^{p}-1\right]_{0}$ dominates a configuration $t_{2} \in\left[2^{p}-1\right]_{0}$ (denoted by $t_{1} \gg t_{2}$ ) iff $B_{t_{1}} \gg B_{t_{2}}$ (recall that $B_{t}$ is the p-dimensional binary representation of $t$ ).

A solution $S^{\prime}$ dominates a solution $S$ (denoted by $S^{\prime} \gg S$ ) iff $\exists$ a bijection $\phi:[n]_{1} \rightarrow[n]_{1}$ such that for any $i \in[n]_{1}, v_{s_{i}^{\prime}} \gg v_{s_{\phi(i)}}$ (in other word, there is a one to one domination between stacks of $S^{\prime}$ and stacks of $S$ ).

A profile $P^{\prime}$ dominates a profile $P$ (denoted by $P^{\prime} \gg P$ ) iff there exists solutions $S^{\prime}$ and $S$ such that $\operatorname{Pr}\left(S^{\prime}\right)=P^{\prime}, \operatorname{Pr}(S)=P$ and $S^{\prime} \gg S$.

Definition 6. For any $i \in[m]_{1}$ and any $t \in\left[2^{p}-1\right]_{0}$, let $b_{t}^{i}$ be the number of vectors of set $V^{i}$ in configuration $t$.

\section{Definition 7 (Graph $G_{P}^{i}$ ).}

Let $P$ be a profile not necessarily feasible. Let $G_{P}^{i}=\left(\left(\Delta_{P}, \Lambda^{i}\right), E_{\gg}\right)$, where $\Lambda^{i}=\left\{\lambda_{t}^{i, l}, 0 \leq t \leq 2^{p}-1,1 \leq l \leq b_{t}^{i}\right\}$, and $\Delta_{P}=\left\{\delta_{t}^{l}, 0 \leq t \leq 2^{p}-1,1 \leq l \leq x_{t}\right\}$. Let us fix a bijection $f: \Delta_{P} \cup \Lambda^{i} \mapsto\left[2^{p}-1\right]_{0}$, that associates to each vertex $\lambda_{t}^{i, l}$ and to each vertex $\delta_{t}^{l}$ the vector in configuration $t . \Lambda^{i}$ (resp. $\Delta_{P}$ ) represents the set of vectors of $V^{i}$ (resp. the demands of profile $P$ ) grouped according to their configurations. Notice that $\left|\Lambda^{i}\right|=\left|\Delta_{P}\right|=n$. Finally, we set $E_{\gg}=\{\{a, b\} \mid a \in$ $\left.\Delta_{P}, b \in \Lambda^{i}, f(a) \ll f(b)\right\}$.

We are now ready to show the following proposition.

Proposition 1. For any profile $P=\left\{x_{0}, \ldots, x_{2^{p}-1}\right\}$,

$\left(\exists P^{\prime}\right.$ feasible, with $\left.P^{\prime} \gg P\right) \Leftrightarrow \forall i \in[m]_{1}, \exists$ a matching of size $n$ in $G_{P}^{i}$

Before starting the proof, notice that the simpler proposition "for any $P, P$ feasible $\Leftrightarrow \forall i \in[m]_{1}$, there is a matching of size $n$ in $G_{P}^{i}$ " does not hold. Indeed, $\Rightarrow$ is correct, but $\Leftarrow$ is not: consider $P$ with $x_{0}=n$ (recall that configuration 0 is the null vector), and an instance with $n m$ "1 vectors" (containing only 1 ). In this case, there is a matching of size $n$ in all the $G_{P}^{i}$, but $P$ is not feasible. This explains the formulation of Proposition 1. An example of the correction formulation is depicted Figure 4.

Proof. Let $P$ be a profile.

$(\Rightarrow)$ Let $P^{\prime}$ be a feasible profile that dominates $P$. Let $S=\left\{s_{1}, \ldots, s_{n}\right\}$ and $S^{\prime}=\left\{s_{1}^{\prime}, \ldots, s_{n}^{\prime}\right\}$ two solutions such that $S^{\prime}$ is feasible, $\operatorname{Pr}(S)=P, \operatorname{Pr}\left(S^{\prime}\right)=P^{\prime}$ (notice that $S$ and $P$ are not necessarily feasible), and $S^{\prime} \gg S$. Without loss of generality, let us assume that $\forall j, s_{j}^{\prime} \gg s_{j}$ (i.e. the bijection $\phi$ of Definition 5 is the identity), and let us assume that for any $j, s_{j}^{\prime}=\left(v_{j}^{1}, \ldots, v_{j}^{m}\right)$. Since $v_{j}^{i} \in s_{j}^{\prime}$, 

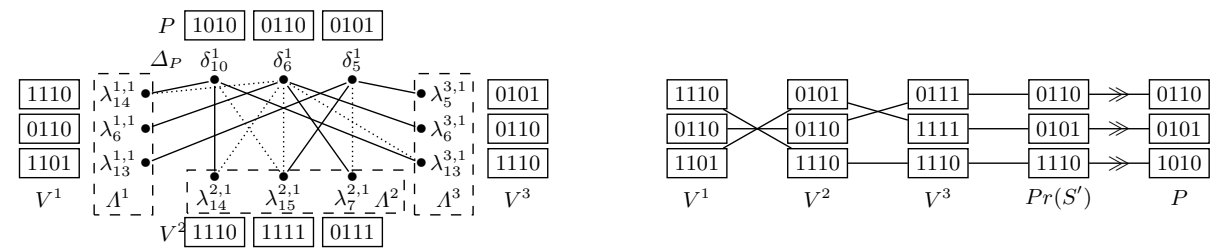

Fig. 4. Illustration of Proposition 1 with $m=n=3$ and $p=4$. Left: The three $G_{P}^{i}$ graphs (edges are depicted by solid and dotted lines), and three matchings (in solid lines) corresponding to $S^{\prime}$. Right: Solution $S^{\prime}$ s.t. $\operatorname{Pr}\left(S^{\prime}\right) \gg P$.

then for any $i$, we know that $v_{j}^{i} \gg s_{j}^{\prime} \gg s_{j}, \forall j \in[n]_{1}$. This implies a matching of size $n$ in all the graphs $G_{P}^{i}$.

$(\Leftarrow)$ Let us suppose that $\forall i \in[m]_{1}$, there is a matching $\mathcal{M}^{i}$ of size $n$ in $G_{P}^{i}$.

W.l.o.g.let us rename $\left\{\delta_{1}, \ldots, \delta_{n}\right\}$ the vertices of $\Delta_{P}$, and $\left\{\lambda_{1}^{i}, \ldots, \lambda_{n}^{i}\right\}$ the vertices of $\Lambda^{i}$ such that for any $i, \mathcal{M}^{i}=\left\{\left\{\lambda_{1}^{i}, \delta_{1}\right\}, \ldots,\left\{\lambda_{n}^{i}, \delta_{n}\right\}\right\}$. This implies $f\left(\lambda_{1}^{i}\right) \gg f\left(\delta_{1}\right), \ldots, f\left(\lambda_{n}^{i}\right) \gg f\left(\delta_{n}\right)$. Let us define $S=\left\{s_{1}, \ldots, s_{n}\right\}$, where $\forall j \in[n]_{1}$, $s_{j}=\left(f\left(\lambda_{j}^{1}\right), \ldots, f\left(\lambda_{j}^{m}\right)\right)$. Notice that for any $j, s_{j} \gg f\left(\delta_{j}\right)$, as all the $f\left(\lambda_{j}^{i}\right) \gg f\left(\delta_{j}\right)$, and combining two vectors $f\left(\lambda_{j}^{i_{1}}\right) \gg f\left(\delta_{j}\right)$ and $f\left(\lambda_{j}^{i_{2}}\right) \gg f\left(\delta_{j}\right)$ creates another vector that dominates $f\left(\delta_{j}\right)$. Thus, $S$ is feasible, and $\operatorname{Pr}(S) \gg P$, and we set $P^{\prime}=\operatorname{Pr}(S)$.

Now, we can use the famous Hall's Theorem to express the existence of a matching in every set.

Theorem 3 (Hall's Theorem). Let $G=\left(\left(V^{1}, V^{2}\right), E\right)$ a bipartite graph with $\left|V^{1}\right|=\left|V^{2}\right|=n$. There is a matching of size $n$ in $G$ iff $\forall \sigma \subseteq V^{1},|\sigma| \leq|\Gamma(\sigma)|$, where $\Gamma(\sigma)=\left\{v_{2} \in V^{2} \mid \exists v_{1} \in \sigma\right.$ such that $\left.\left\{v_{1}, v_{2}\right\} \in E\right\}$.

Remark 1. Notice that we cannot use Hall's Theorem directly on graphs $G_{P}^{i}$, as we would have to add the $2^{n}$ constraints of the form $\forall S \subseteq V^{i}$. However, we will reduce the number of constraints to a function $f(p)$ by exploiting the particular structure of $G_{P}^{i}$.

Proposition 2 (Matching in $G_{P}^{i}$ ).

$\forall i \in[m]_{1}, \forall P=\left\{x_{0}, \ldots, x_{2^{p}-1}\right\}$ :

$\left(\forall \sigma \subseteq \Delta_{P},|\sigma| \leq|\Gamma(\sigma)|\right) \Leftrightarrow\left(\forall \sigma_{c f g} \subseteq\left[2^{p}-1\right]_{0}, \sum_{t \in \sigma_{c f g}} x_{t} \leq \sum_{t \in \text { dom }\left(\sigma_{c f g}\right)} b_{t}^{i}\right)$ where $\operatorname{dom}\left(\sigma_{c f g}\right)=\left\{t^{\prime} \mid \exists t \in \sigma_{c f g}\right.$ such that $\left.t^{\prime} \gg t\right\}$ is the set of configurations that dominate $\sigma_{c f g}$.

Proof. $(\Rightarrow)$ Let $\sigma_{c f g}=\left\{t_{1}, \ldots, t_{\alpha}\right\}$. Let $\sigma=\left\{\delta_{t_{i}}^{l}, 1 \leq i \leq \alpha, 1 \leq l \leq x_{t_{i}}\right\}$ be the vertices of $\Delta_{P}$ corresponding to the demands in $\sigma_{c f g}$. Observe that $\sum_{t \in \sigma_{c f g}} x_{t}=$ $|\sigma|$. Notice also that $\Gamma(\sigma)=\left\{\lambda_{t}^{i, l}, t \in \operatorname{dom}(\sigma), 1 \leq l \leq b_{t}^{i}\right\}$ by construction. Thus, $|\sigma| \leq|\Gamma(\sigma)|$ implies $\sum_{t \in \sigma_{c f g}} x_{t} \leq \sum_{t \in d o m\left(\sigma_{c f g}\right)} b_{t}^{i}$.

$(\Leftarrow)$ Let $\sigma \subseteq \Delta_{P} . \forall t \in\left[2^{p}-1\right]_{0}$, let $X_{t}=\left\{\delta_{t}^{l}, 1 \leq l \leq x_{t}\right\}$, let $\sigma_{t}=\sigma \bigcap X_{t}$. Let $\sigma_{\text {cfg }}=\left\{t_{1}, \ldots, t_{\alpha}\right\}=\left\{t \mid \sigma_{t} \neq \emptyset\right\}$. Let $X=\bigcup_{t \in \sigma_{\text {cfg }}}\left\{X_{t}\right\}$. Notice that $|\sigma| \leq|X|=$ $\sum_{t \in \sigma_{c f g}} x_{t}$.

Let us first prove that $\Gamma(\sigma)=\Gamma(X) . \Gamma(\sigma) \subseteq \Gamma(X)$ is obvious. Now, if there is a $\lambda_{t^{\prime}}^{i, l^{\prime}} \in \Gamma(X)$, it means that there is a $t \in \sigma_{c f g}$ such that $\lambda_{t^{\prime}}^{i, l^{\prime}} \in \Gamma\left(X_{t}\right)$, and 
thus there exists $l$ such that $\left\{\delta_{t}^{l}, \lambda_{t^{\prime}}^{i, l^{\prime}}\right\} \in E$ (which implies that $t^{\prime} \gg t$ ). As $\sigma_{t} \neq \emptyset$, there exists $l^{\prime}$ such that $\delta_{t}^{l^{\prime}} \in \sigma_{t}$, and $\left\{\delta_{t}^{l^{\prime}}, \lambda_{t^{\prime}}^{i, l^{\prime}}\right\} \in E$ as $t^{\prime} \gg t$.

Finally, the hypothesis with our set $\sigma_{c f g}$ leads to

$$
|\sigma| \leq|X|=\sum_{t \in \sigma_{c f g}} x_{t} \leq \sum_{t \in \operatorname{dom}\left(\sigma_{c f g}\right)} b_{t}^{i}=|\Gamma(X)|=|\Gamma(\sigma)|
$$

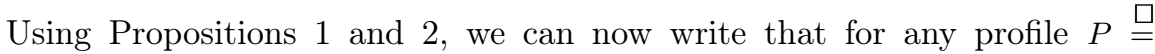
$\left\{x_{0}, \ldots, x_{2^{p}-1}\right\}$ :

$$
\exists P^{\prime} \text { feasible, with } P^{\prime} \gg P \Leftrightarrow \forall i, \forall \sigma_{c f g} \subseteq\left[2^{p}-1\right]_{0}, \sum_{t \in \sigma_{c f g}} x_{t} \leq \sum_{t \in \operatorname{dom}\left(\sigma_{c f g}\right)} b_{t}^{i} .
$$

Thus, we use now the following ILP to describe the $\max \sum 1$ problem:

$$
\begin{array}{ll}
\max & \sum_{t=0}^{2^{p}-1} x_{t} c_{t} \\
\text { subject to } & \forall i \in[m]_{1}: \forall \sigma_{c f g} \subseteq\left[2^{p}-1\right]_{0}, \sum_{t \in \sigma_{c f g}} x_{t} \leq \sum_{t \in \operatorname{dom}\left(\sigma_{c f g}\right)} b_{t}^{i} \\
& \forall t \in\left[2^{p}-1\right]_{0}, x_{t} \in \mathbb{N}
\end{array}
$$

This linear program has $2^{p}$ variables and $\left(m 2^{2^{p}}+2^{p}\right)$ constraints. Thus, we can solve it using [8] in time $f(p)$ poly $(n+m)$, we get that $\max \sum 1$ and $\min \sum 0$ are FPT parameterized by $p$. Using Property 2 this ILP leads to a $\frac{p}{r}$-approximation algorithm for max $\sum 1$ running in time $f(r)$ poly $(n+m+p)$.

\section{Conclusion}

In this article, we established that $\max \sum 1$ is $\mathcal{O}\left(m^{1-\varepsilon}\right)$ and $\mathcal{O}\left(p^{1-\varepsilon}\right)$ non-approximable for $n=2$. On the positive side, we provided a FPT algorithm for max $\sum 1$ leading to a $\frac{p}{r}$-approximation algorithm running in $\mathcal{O}(f(r)$ poly $(m+n+p))$, which is the best we can hope for. The existence of a $\rho(m)$-approximation (typically $m$ ) algorithm remains open.

\section{References}

1. P. Crescenzi, V. Kann, R. Silvestri, and L. Trevisan. Structure in approximation classes. SIAM Journal on Computing, 28(5):1759-1782, 1999.

2. M. Cygan. Improved approximation for 3-dimensional matching via bounded pathwidth local search. In Foundations of Computer Science (FOCS), 2013 IEEE 54th Annual Symposium on, pages 509-518. IEEE, 2013.

3. T. Dokka, M. Bougeret, V. Boudet, R. Giroudeau, and F. CR Spieksma. Approximation algorithms for the wafer to wafer integration problem. In Approximation and Online Algorithms (WAOA), pages 286-297. Springer, 2013.

4. T. Dokka, Y. Crama, and F. C.R. Spieksma. Multi-dimensional vector assignment problems. Discrete Optimization, 14:111-125, 2014.

5. G Duvillié, M Bougeret, V Boudet, T Dokka, and R Giroudeau. On the complexity of Wafer-to-Wafer Integration. Research report, Lirmm ; UM II montpellier, Faculté des Sciences et Techniques du Languedoc, January 2015. HAL id:lirmm01110027. 
6. E. Hazan, S. Safra, and O. Schwartz. On the complexity of approximating kdimensional matching. In Approximation, Randomization, and Combinatorial Optimization.. Algorithms and Techniques, pages 83-97. Springer, 2003.

7. S. Kratsch. On polynomial kernels for integer linear programs: Covering, packing and feasibility. In Algorithms-ESA 2013, pages 647-658. Springer, 2013.

8. H. W. Lenstra Jr. Integer programming with a fixed number of variables. Mathematics of operations research, 8(4):538-548, 1983.

9. R. Niedermeier. Invitation to fixed-parameter algorithms. 2006.

10. S. Reda, G. Smith, and L. Smith. Maximizing the functional yield of wafer-towafer 3-d integration. IEEE Transactions on Very Large Scale Integration (VLSI) Systems, 17(9):1357-1362, 2009.

11. D. P. Williamson and D. B. Shmoys. The design of approximation algorithms. Cambridge University Press, 2011.

12. D. Zuckerman. Linear degree extractors and the inapproximability of max clique and chromatic number. In Proceedings of the thirty-eighth annual ACM symposium on Theory of computing, pages 681-690. ACM, 2006. 